\title{
Políticas de la Regiones Educativas en Puerto Rico: Hacia la Uniformidad de los Procesos Gerenciales
}

\author{
Home Education Policies in Puerto Rico: Towards the \\ Uniformity of Management Processes
}

Glenda L. García Bonilla *

Pontificia Universidad Católica de Puerto Rico

\begin{abstract}
En los últimos años, la educación puertorriqueña ha evolucionado y con ello ha tomado auge la modalidad de educación en el hogar. La presente investigación tiene como propósito determinar cuáles son las políticas administrativas y gerenciales por las cuales se rigen las organizaciones que agrupan a las familias que educan a sus hijos en el hogar en Puerto Rico. La investigación fue de carácter cualitativo con un enfoque etnográfico-estudio de caso. Para la misma participaron siete directivos y dieciséis administradoras del hogar. Los datos fueron recopilados mediante dos instrumentos de entrevista diseñados por la investigadora; uno dirigido a los directivos y otro a los administradores del hogar. La información sociodemográfica fue analizada mediante estadística descriptiva. Se utilizó la técnica de triangulación para determinar la divergencia y convergencia de los datos recopilados. Se encontró que las familias que educan en el hogar en Puerto Rico tienen un perfil variado (académico, religioso y socioeconómico), las madres se dedican a enseñar en el hogar y utilizan diversas metodologías de enseñanza para educar a sus hijos en el hogar. Según los resultados, se concluyó que no existen uniformidad en las políticas administrativas y gerenciales de las organizaciones de educación en el hogar, el currículo es atractivo y flexible, la individualidad, valores morales y religiosos han creado que esta modalidad sea adopta por muchas familias puertorriqueñas.
\end{abstract}

Descriptores: Liderazgo, Gerente, Educación, Educación básica, Política educacional.

In recent years, Puerto Rican education has evolved and with this the home education modality has taken hold. The purpose of this research is to determine the administrative and management policies that govern the organizations that bring together the families that educate their children at home in Puerto Rico. The research used a qualitative focus, ethnographic, and case study approach. For the same participated seven managers and sixteen household. The data were collected through two interview instruments designed by the researcher; one for managers and the other for administrators of the household. The sociodemographic information was analyzed using descriptive statistics. The triangulation technique was used to determine the divergence and convergence of the data collected. It was found that families that practice home-schooling in Puerto Rico have a varied profile (academic, religious and socioeconomic), mothers are dedicated to teaching at home and use various teaching methodologies to educate their children at home. According to the results, it was concluded that there is no uniformity in the administrative and management policies of Home Education Organizations, the curriculum is attractive and flexible; individuality, moral and religious values have created that this modality is adopted by many Puerto Rican families.

Keywords: Leadership, Managers, Education, Basic education, Educational policy.

*Contacto: glendagarcia@pucpr.edu

ISSN: $1696-4713$

www.rinace.net/reice/

revistas.uam.es/reice
Recibido: $\quad 20$ de julio 2017

$1^{\text {a }}$ Evaluación: 13 de agosto 2017

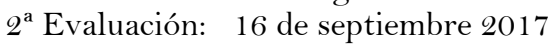

Aceptado: $\quad 20$ de septiembre 2017 


\section{Introducción}

La modalidad de educar en el hogar ha tenido gran aceptación y auge en el contexto educativo puertorriqueño (Acevedo, 2012). Bajo esta modalidad los padres se convierten en maestros de sus propios hijos o seleccionan tutores que les ofrecen clases privadas a los mismos. El término educación en el hogar se define como aquella acción en que los padres seleccionen un programa, método o currículo de estudio para realizarlo en su hogar, donde estos destinan un espacio y un tiempo específico para estudiar y cumplir con los requisitos de la educación en el hogar de sus hijos (Holt y Farenga, 2003). De acuerdo con Rivero (2008) algunas razones para utilizar esta modalidad pueden ser de carácter económico, social, religioso o de otra índole.

Desde finales del 1999 en el departamento de estado de Puerto Rico se han inscrito cuatro organizaciones de educación en el hogar. Estas organizaciones son la Asociación de Educadores en el Hogar Puertorriqueño Adventista, The Caribbean Center of Home Education Resources, la Casa Escuela Puerto Rico, y la Asociación Hogares-Escuela Cristianas en Puerto Rico. Cada organización ha desarrollado políticas y reglamentos particulares para sus participantes. Esto refleja la falta de uniformidad en las normativas, procedimientos y líneas de intervención para la enseñanza bajo la modalidad de educación en el hogar. Bajo la presunción de que no hay políticas uniformes establecidas por las organizaciones de educación en el hogar en Puerto Rico que permitan unificar los principios y propósitos de esta modalidad de estudio, se desarrolló la investigación titulada "Políticas de la educación en el hogar en Puerto Rico: Hacia la uniformidad de los procesos gerenciales".

La investigadora recopiló información sobre las políticas de las diferentes organizaciones que educan en el hogar en Puerto Rico con el propósito de describir las prácticas y modelos educativos que utilizan. Se utilizó como población a los miembros de las cuatro organizaciones de educación en el hogar inscritas en el Departamento de Estado de Puerto Rico. Los participantes fueron dos grupos focales, una Junta Directiva y la presidenta de una organización que educan en el hogar y 16 padres que pertenecen a las organizaciones bajo estudio. La investigación fue cualitativa de carácter etnográfico con un enfoque de estudio de caso. La misma analiza si existe uniformidad en los procesos administrativos y gerenciales de las organizaciones de regiones educativas en Puerto Rico. Se conocerán las metodologías de enseñanza de las familias que educan en el hogar y las razones por las cuales los padres deciden educar en casa.

\section{Revisión de la literatura}

En la década de los setenta y principios de los ochenta llegaron a Puerto Rico militares estadounidenses y con ellos sus esposas. Se establecieron en la Base Naval Rooselvet Roads, en el área este de Puerto Rico y con ello se reinició la educación en el hogar en la isla (Sánchez, 2013). La diferencia cultural y el idioma inglés obligaron a las esposas de los militares a educar a sus hijos en las bases militares. Esta modalidad educativa comenzó a propagarse en las familias de Humacao y pueblos cercanos.

Según Sánchez (2013), en la Base Buchanan en Guaynabo se comenzaron a organizar las esposas de los militares con la integración de familias puertorriqueñas. A principios de la década de las noventa, las familias de los militares fueron relocalizadas en Estados Unidos dejando el grupo que formaron de educación en el hogar en manos de las familias 
puertorriqueñas. Mientras, las familias puertorriqueñas se organizaron y decidieron salvar esta modalidad educativa puertorriqueña.

En el 1999 se fundaron y quedaron establecidas en el Departamento de Estado de Puerto Rico las organizaciones que educan en el hogar The Caribbean Center of Home Education Resources y Asociación de Educadores en el Hogar Puertorriqueño Adventista (Sánchez, 2013). En el año 2000 la educación en el hogar en Puerto Rico comenzó a tener un auge significativo; los padres que educan en el hogar comenzaron a organizarse como un grupo privado (Sánchez, 2013). Para el año 2002 comenzó la Legislatura a preocuparse por legislar la educación en el hogar debido al aumento de los estudiantes educados en el hogar en Puerto Rico. El Proyecto 3048 del ex representante José Javier García Cabán (2002) tuvo la intención de crear un programa de enseñanza en el hogar. Sin embargo, Michael Smith, presidente de la Home School Legal Defense Association (2003) aseguró que ese proyecto era el peor que se había presentado en la Legislatura haciendo referencia a la falta de información y criterios para evaluar la educación en el hogar, ya que no existían regulaciones legales.

En el 2006 la Cámara de Representantes hizo su segundo intento de regular la educación en el hogar con el Proyecto 2754 de la ex representante Albita Rivera. Su intención fue establecer la educación en el hogar como una institución educativa no tradicional. La falta de estructura, información y penalidad a los padres que educan en el hogar provocó que la Coalición Homeschooler derogara el proyecto (Sánchez, 2013).

En el año 2011 la Legislatura de Puerto Rico realizó un tercer intento para legislar la educación en el hogar con el Proyecto 2110 del senador Thomas Rivera Schatz (Sánchez, 2013). Este tuvo como fin adoptar la Ley del Derecho de la Educación y establecer cuáles eran los derechos y responsabilidades de los padres que optaran por la educación en el hogar. Rivera Schatz (2011) hizo una comparación de la educación en el hogar en Puerto Rico con la de otros países, haciendo énfasis en la responsabilidad de los padres en el método de educar a sus hijos y estableciendo penalidades si incumplían con las normas establecidas para esta metodología educativa. Sin embargo, la Coalición Homeschooler no estuvo de acuerdo con los puntos establecidos en el proyecto de ley y dejándose llevar por la Carta de Derechos de 1952 Sección II, Artículo V. El Proyecto fue derogado.

El cuarto intento de la legislatura para legislar la educación en el hogar ocurrió en el 2013 con el Proyecto 793 del senador Cirilo Tirado Rivera quien explicó cómo la educación en el hogar ha sido clasificada en diferentes países y bajo qué condiciones con la finalidad de determinar la cantidad de niños educados en el hogar y las instrucciones impartidas por sus padres para obtener datos estadísticos. El senador señaló que según la Constitución de Puerto Rico (1952) "es un deber ineludible que tiene la legislatura de salvaguardar las libertades que defienden la educación del hogar” (Sección 5, Artículo II). El $80 \%$ de las universidades en Estados Unidos, incluyendo la Universidad de Puerto Rico y la Universidad Politécnica han adaptado sus políticas institucionales para aceptar estudiantes que han sido educados en el hogar. Tirado Rivera (2013) señaló que la escuela en casa responde a la idea de no child left behind ya que tiene como fin individualizar la educación y que cada educando pueda progresar a tenor con sus capacidades. Sin embargo, Mike Smith, presidente de HSLD crea una oposición ante este proyecto de ley porque lo que busca es identificar a los padres y sus hijos con el propósito de darle seguimiento. Smith añadió que el gobierno de Puerto Rico no confía 


\section{G. L. García Bonilla}

en los padres que educan a sus hijos en casa y lo que busca es regular la modalidad por lo cual incitó a las familias que educan en el hogar a realizar una manifestación en el Capitolio en contra de esta legislación. El proyecto fue derogado.

El ex secretario de Educación de Puerto Rico, el Prof. Rafael Román, el 27 de julio de 2015 envió un comunicado sobre los procedimientos que debe seguir un estudiante que deseen ingresar al sistema de educación pública y provienen de la educación en el hogar. En el mismo estipuló que todo estudiante que desee ingresar al sistema público de educación será enviado a la Unidad de Estudios de Equivalencia a nivel Central o en la región educativa a la cual pertenece para completar el proceso de equivalencia.

Román (2015) añadió que, para evaluar estos casos, el estudiante deberá someter los siguientes documentos: a) un portafolio por asignatura de todos los trabajos que realizo durante la educación en el hogar, b) lista de libros utilizados por grado y año escolar, c) currículo o temas discutidos en cada grado escolar, d) trascripción de créditos del último grado aprobado en una escuela acreditada, y e) información de la escuela donde el estudiante será matriculado. Cada nivel cuenta con el procedimiento a seguir para cada uno de los estudiantes. Román (2015) enfatizó que si un estudiante deseaba darse de baja del sistema de enseñanza pública para que sus padres lo eduquen en el hogar conlleva de un procedimiento a seguir. Primeramente, el Comité de Retención Escolar (CORE) mostrará evidencia de todas las gestiones realizadas para retener el estudiante en la escuela según lo dispone la carta circular 13-2013-2014 sobre la Política Pública para establecer el procedimiento a seguir en la implementación de las normas de retención escolar en el Departamento de Educación. El segundo paso, el director de la escuela y el personal de apoyo deberán realizar una reunión y llenar una minuta de la baja del estudiante y el cambio a ser educado en casa. El tercer paso, es llenar el formulario Informe oficial de movimiento de matrícula vigente con la codificación B13, si la transacción ocurre del 1 de agosto al 31 de mayo del año en curso, de lo contrario la codificación será V14. Por último, el director le informará al ayudante especial del distrito y al Departamento de la Familia de su región y llevando evidencia de las acciones y minuta del CORE.

Esta investigación se enmarca en la teoría de sistemas ecológicos de Urie Bronfenbrenner y en la teoría de sistemas de Lodwing Von Bertanlanffy. De acuerdo con Bronfenbrenner (1979), el desarrollo del ser humano comprende los entornos en que viven las personas a través de su vida. Este autor definió el término desarrollo como un proceso complejo en el que se ponen en juego la cantidad de factores familiares, religiosos, en el lugar de trabajo y que no pueden ser reducidos. La teoría de sistemas ecológicos definió como una forma de explicar las interacciones del niño en su medio ambiente y como ese entorno afecta su crecimiento y desarrollo. Aunque esta presenta cinco sistemas que interactúan en el desarrollo humano (microsistema, mesosistema, exosistema, macrosistema y cronosistema), para fines de esta investigación se atemperó a tres sistemas: microsistema, mesosistema y macrosistema. Desde la perspectiva de la educación en el hogar, la cultura familiar es donde se desarrolla la enseñanza y organización familiar para establecer las líneas y estrategias de enseñanza. Según la teoría de Bronfenbrenner (1979) el microsistema hace referencia a la familia, a los grupos y a los pares para determinar las relaciones interpersonales del individuo dentro de un entorno. Por otro lado, el mesosistema se refiere al desarrollo del individuo dentro de dos o más entornos, relación familia-grupo-trabajo. El macrosistema se refiere a cada organización que educa en el hogar y que posee sus propias políticas, creencias, valores, 
políticas y reglamentos. Dentro de cada organización existen los diferentes microsistemas, los cuales componen el macrosistema. Es en el macrosistema donde los niños educados en el hogar y sus familias se organizan como grupo y comparten con otros grupos experiencias, metodologías de enseñanza, técnicas y valores formando un sistema más complejo con un fin en común, educar en el hogar.

Por otro lado, la teoría ecología de Bronfenbrenner se relaciona con la teoría de sistemas de Lodwing Von Bertalanffy. Von Bertalanffy (1968) definió el término sistema como un conjunto de elementos en estado de interacción. Según este autor, un sistema es un conjunto de partes las cuales deben interactuar para que el sistema sea permanente. Existen diferentes tipos de sistemas, entre los cuales se pueden mencionar: familia, grupo, sistema social y organizaciones. Las características del sistema, de acuerdo con este autor, son: a) la acción recíproca en la cual todos los elementos deben actuar recíprocamente para que el sistema funcione, b) depende de la presencia o ausencia de todas las partes, sino el sistema deja de funcionar, c) las partes del sistema cada vez son mayores por eso se dividen en subsistemas, y d) los sistemas son diferentes y complejos.

En Puerto Rico existen organizaciones y grupos de apoyo con el fin de brindarles ayuda a los administradores del hogar cuando seleccionan la modalidad de la educación en el hogar para sus hijos. Estas organizaciones son sistemas abiertos fundamentados en los insumos, tales como: la familia, el niño que es educado en el hogar y las diferentes metodologías. El fin de cada organización es producir resultados para la población a la cual está determinada (educación en el hogar) (Von Bertalanffy, 1968). La educación en el hogar es un sistema abierto con factores determinantes que lo hacen dividir en subgrupos para que todos los elementos que componen el sistema puedan trabajar recíprocamente.

Las preguntas que se establecieron en la presente investigación fueron: ¿Cuál es el perfil de las familias que educan a sus hijos en el hogar? ¿Cuáles son las políticas administrativas y gerenciales de las diferentes organizaciones que agrupan a las familias que educan en el hogar en Puerto Rico? ¿Cómo comparan las políticas administrativas y gerenciales de las organizaciones que agrupan a las familias que educan en el hogar en Puerto Rico? ¿Cuáles son las diferentes metodologías de enseñanza que son utilizadas por las cuatro organizaciones que agrupan a las familias que educan en el hogar en Puerto Rico?

Los objetivos establecidos para desarrollar la investigación fueron:

- Analizar el perfil de las familias que educan en el hogar.

- Describir las políticas administrativas y gerenciales de las organizaciones que agrupan a las familias que educan en el hogar en Puerto Rico.

- Comparar las políticas administrativas y gerenciales de las organizaciones que agrupan a las familias que educan en el hogar en Puerto Rico.

- Describir las metodologías de enseñanza en las cuatro organizaciones que agrupan a las familias que educan a sus hijos en el hogar. 


\section{Método}

\section{Enfoque metodológico}

El diseño de la investigación fue de carácter cualitativo de estudio de caso-etnográfico y se fundamentó en una descripción detallada de las políticas administrativas y gerenciales de la educación en el hogar desde la perspectiva de siete directivos de dos organizaciones que educan en el hogar y de 16 padres que pertenecen a las cuatro organizaciones bajo estudio. De acuerdo con Creswell (2009), la investigación cualitativa requiere de un plan de acción de campo para recopilar la información. Este propuso que se logre un acercamiento con los participantes con el propósito de estudiar los rasgos culturales, valores y creencias. El método etnográfico se define como la forma de indagar y examinar lo que un determinado grupo hace, vive e interactúa en un ambiente dado (Cabrera, 2010). La etnografía requiere que la investigadora viva y comparta dentro del grupo, y que desde ese lugar se pueda analizar e interpretar como los miembros del grupo le dan sentido al mundo. El diseño cualitativo etnográfico, permite el estudio de diferentes culturas en un tiempo determinado, lo que hace que sea idóneo para la realización de esta investigación (Cabrera, 2010).

El grupo focal de la organización A estuvo compuesto por seis miembros de la directiva. De la organización B solo se entrevistó por correo electrónico a un miembro de la directiva. A cada organización se le proveyó información relacionada con el propósito y el procedimiento de la investigación y se acordó el lugar y fecha para llevar a cabo las entrevistas. La selección de los administradores del hogar se hizo a base de las siguientes características: educaban en el hogar y estaban afiliados a una organización de educación en el hogar inscrita en el Departamento de Estado de Puerto Rico. Se seleccionaron seis administradores del hogar de la organización A, un administrador del hogar de la organización B, un administrador del hogar de la organización C y ocho administradores del hogar de la organización D.

\section{Contexto}

La presente investigación estuvo dirigida a analizar e interpretar experiencias y valores, entre otros elementos presentes, a través de las entrevistas realizadas a los grupos focales de los directivos pertenecientes a dos de las organizaciones A y B de educación en el hogar y a los 16 padres participantes.

Esta investigación se llevó a cabo en ambientes naturales seleccionados por los directivos y padres, en coordinación con la investigadora, con el fin de identificar los patrones culturales de la modalidad de educación en el hogar. Los ambientes naturales fueron: dos parques, una iglesia y vía correo electrónico.

\section{Participantes}

La población para esta investigación estuvo constituida por los directivos de las cuatro organizaciones que educan en el hogar inscritas en el Departamento de Estado de Puerto Rico y los administradores del hogar (padres) que pertenecen a las mismas. La selección de los participantes es determinante en una investigación cualitativa. En esta investigación los participantes fueron seleccionados a base de las siguientes características: educan en el hogar y pertenecen a una de las organizaciones inscritas en el Departamento del Estado. Los participantes fueron: seis directivos de la organización A, un directivo de la organización B. No hubo participación de los directivos de las 
organizaciones C y D. Una parte de los participantes conformó un grupo focal de la organización A y un directivo de la organización B que operan en Puerto Rico.

El segundo grupo de participantes fueron los administradores del hogar (padres). Participaron 16 padres pertenecientes a las cuatro organizaciones que educan en el hogar. Tres administradores del hogar completaron grados asociados, diez administradores del hogar tenían preparación académica de bachillerato, dos con grado de maestría y una administradora del hogar con un grado doctoral. Estas muestras no fueron sometidas a procedimientos rigurosos de muestreo, lo cual significa que los datos obtenidos de esta investigación aplicarán solamente a la población de las cuatro organizaciones de educación en el hogar participantes.

Cuadro 1. Perfil sociodemográfico de los directivos y administradores del hogar

\begin{tabular}{|c|c|c|c|c|c|c|c|c|}
\hline \multicolumn{3}{|c|}{ DiREctivos } & \multicolumn{6}{|c|}{ ADMINISTRADORES DEL HOGAR } \\
\hline Características & $\mathbf{N}$ & $\%$ & Características & $\mathbf{N}$ & $\%$ & Características & $\mathbf{N}$ & $\%$ \\
\hline Sexo & & & Sexo & \multicolumn{5}{|c|}{ Aspecto socioeconómico } \\
\hline Femenino & 4 & 57,14 & Femenino & 16 & 1 & Padre trabaja & 5 & 31,25 \\
\hline Masculino & 3 & 42,86 & Masculino & $\mathrm{O}$ & o & Madre trabaja & 4 & 25,00 \\
\hline Edad & & & Edad & & & Trabajan ambos & 3 & 18,75 \\
\hline $30-40$ & 2 & 28,57 & $30-40$ & 9 & 56,25 & Desempleados & 2 & 12,50 \\
\hline $41-50$ & 2 & 28,57 & $41-50$ & 5 & 31,25 & $\mathrm{NC}$ & 2 & 12,50 \\
\hline $51-60$ & 1 & 14,29 & $51-60$ & 1 & 6,25 & Región educativa & & \\
\hline $61-70$ & 1 & 14,29 & $61-70$ & 1 & 6,25 & Caguas & 7 & 43,75 \\
\hline 71 o más & 1 & 14,29 & \multicolumn{2}{|c|}{ Preparación académica } & & San Juan & 3 & 18,75 \\
\hline Preparación académic & & & Grado Asociado & 3 & 18,75 & Humacao & 4 & 25,00 \\
\hline Escuela superior & 1 & 14,29 & Bachillerato & 10 & 62,50 & Bayamón & 2 & 12,50 \\
\hline Grado asociado & $\mathrm{O}$ & 0,00 & Maestría & 2 & 12,50 & \multicolumn{3}{|c|}{ Núm. hijos educados en casa } \\
\hline Bachillerato & 4 & 57,14 & Doctorado & 1 & 6,25 & $1-3$ & 15 & 94,00 \\
\hline Maestría & 2 & 28,57 & \multicolumn{2}{|c|}{ Años educando en casa } & & $4-6$ & 1 & 6,00 \\
\hline Región educativa & & & $1-3$ & 8 & 50,00 & \multicolumn{2}{|c|}{ Religión que practican } & \\
\hline Caguas & 4 & 57,14 & $4-6$ & 2 & 12,50 & Adventista & 6 & 37,50 \\
\hline San Juan & 1 & 14,29 & $7-9$ & 3 & 18,75 & Pentecostal & 2 & 12,50 \\
\hline Humacao & 2 & 28,57 & $10-12$ & 1 & 6,25 & Protestante & 1 & 6,25 \\
\hline Núm. hijos educados & cas & & $13-15$ & 2 & 12,50 & Mormones & 1 & 6,25 \\
\hline $1-3$ & 6 & 85,72 & & & & Católica & 4 & 25,00 \\
\hline $4-6$ & 1 & 14,28 & & & & $\mathrm{NC}$ & 2 & 12,50 \\
\hline
\end{tabular}

Nota: NC, No contesta.

Fuente: Elaboración propia.

En el cuadro 1 se puede apreciar que el $57 \%$ de los directivos de las organizaciones de educación en el hogar eran féminas. El $58 \%$ de los directivos está entre las edades de 30 a 50 años. En cuanto a la preparación académica, el $86 \%$ tiene preparación académica desde bachillerato hasta maestría. Más de la mitad (57\%) de los directivos pertenecen a la región educativa de Caguas. La mayoría (86\%) ha educado de uno a tres hijos en el hogar. También se observa que el $100 \%$ de los administradores del hogar participantes eran féminas. El $56,25 \%$ de las participantes está entre las edades de 30 a 40 años. El $62,50 \%$ tiene preparación académica de bachillerato. La mitad $(50 \%)$ de las participantes llevan de uno a tres años educando en el hogar. El 56,25\% indicó que solo el padre o la madre trabajan fuera del hogar. En cuanto a la cantidad de hijos educados en el hogar, 15 madres (94\%) indicaron de uno a tres hijos. El 43,75\% indicó que pertenecen a la 


\section{G. L. García Bonilla}

región educativa de Caguas. En cuanto a la religión que practican, las que obtuvieron los mayores porcentajes fueron la adventista $(37,50 \%)$ y la católica $(25 \%)$.

\section{Instrumentos}

En la recopilación de la información necesaria para la investigación "Políticas de la educación en el hogar en Puerto Rico: Hacia la uniformidad de los procesos gerenciales" se utilizaron dos instrumentos que consistieron de entrevistas focalizadas diseñadas por la investigadora. El primer instrumento estuvo dirigido al grupo focal compuesto por los directivos de las organizaciones de educación en el hogar que operan en Puerto Rico con el fin de establecer su perfil y conocer sus políticas y reglamentos. El instrumento constó con 11 preguntas fundamentales que abrieron paso a nuevas interrogantes. Estas entrevistas se grabaron en audio y luego fueron transcritas.

El segundo instrumento se administró a los administradores del hogar (padres) y con el mismo se pretendió establecer el perfil de los padres que educan en el hogar. La entrevista constó de 22 preguntas que ayudaron a conocer, entre otros, el conocimiento de las políticas y reglamentos de la organización de educación en el hogar a la que pertenecen, los motivos para educar en el hogar y las metodologías educativas utilizadas. Además, se analizaron los reglamentos operacionales de las cuatro organizaciones de regiones educativas participantes en la investigación. Con el propósito de comparar los procesos gerenciales de cada organización.

Las investigaciones cualitativas dependen de la recopilación e interpretación de los datos del investigador (Lucca y Berríos, 2003). Es por esta razón que los dos instrumentos que se utilizaron para realizar las entrevistas se sometieron a un proceso de validación en el que participaron profesionales con conocimiento en investigación cualitativa y en educación en el hogar. Los expertos que validaron los instrumentos fueron: dos expertos con conocimientos en educación en el hogar en Puerto Rico y un experto con conocimiento en investigación cualitativa. Según Creswell (2009) los expertos tienen como función evaluar las preguntas de los instrumentos, de manera que estén claras, completas y permitan recopilar la información necesaria para contestar las preguntas de la investigación. La figura 1 representa el esquema de las etapas del trabajo de campo que condujeron a esta investigación.

\section{Análisis de datos}

En un estudio cualitativo la recolección y análisis de datos se llevan a cabo de forma simultánea. Creswell (2009) afirma que, en el análisis de datos, primeramente, se clasifican las entrevistas, las personas y los eventos para determinar las características comunes entre ellos. El segundo paso es codificar los datos mediante categorías, describir los patrones y temas más frecuentes. En tercer lugar, se revisan las entrevistas y se organizan por categorías y/o códigos, y, por último, se transcriben.

Una vez realizadas las entrevistas al grupo focal de los directivos y de los administradores del hogar, se analizaron los datos sociodemográficos mediante estadística descriptiva. Las entrevistas (cuadro 2) fueron estructuradas y grabadas y las observaciones se transcribieron. Los datos recopilados se analizaron mediante una prueba de triangulación. La prueba de triangulación consiste en contrastar las descripciones de los informantes de las diferentes organizaciones con el objetivo de determinar la credibilidad de la información (Bisquerra, 2002). Además de la prueba de triangulación se realizó un contraste de las respuestas obtenidas de los participantes con 
las normativas de la organización. Con uno de los directivos de la organización A se realizó un cotejo de participante para verificar la credibilidad obtenida en la investigación.

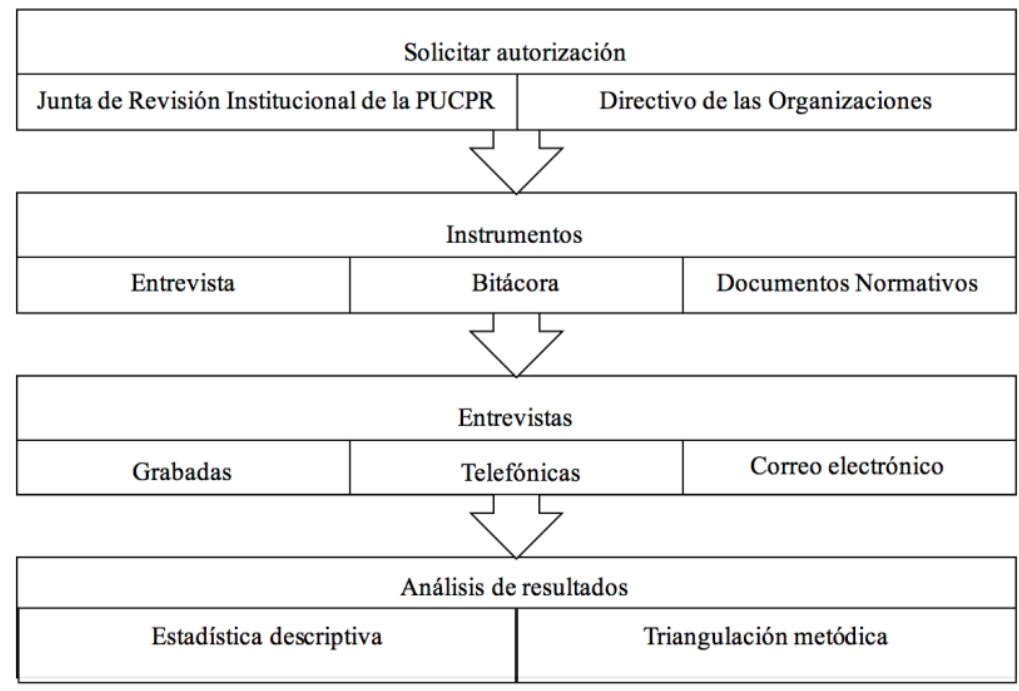

Figura 1. Resumen del procedimiento Fuente: Elaboración propia.

\section{Resultados}

El propósito de esta investigación fue auscultar la uniformidad de las políticas administrativas y gerenciales de las organizaciones que educan en el hogar en Puerto Rico. Para esto, la investigadora diseñó dos entrevistas. La primera entrevista estuvo dirigida a los directivos de las organizaciones de educación en el hogar para conocer sus políticas y reglamentos, identificar un perfil general de las familias que pertenecen a su organización y conocer las metodologías y técnicas de enseñanza que utilizan los padres para desarrollar sus clases. La segunda entrevista estuvo dirigida a los administradores del hogar con el fin de determinar si ellos conocían las políticas administrativas y gerenciales de las organizaciones de educación en el hogar a las cuales pertenecen, identificar el perfil de las familias que educan en el hogar y conocer qué estrategias y metodologías académicas que utilizan para desarrollar sus clases diariamente.

A continuación, el cuadro 3 y el cuadro 4 presentan las respuestas a cada una de las preguntas que guiaron esta investigación. En la misma participaron los directivos de dos de las organizaciones bajo estudio y 16 administradores del hogar pertenecientes a las cuatro organizaciones participantes de la investigación. Para mantener la confidencialidad de los participantes se utilizaron las siguientes codificaciones: AD1 (organización A, Directivos 1), BD1 (organización B, Directivos 1), AA1 al AA6 (organización A, Administradoras del hogar 1 al 6), BA1 al BA8 (organización B, Administradora del hogar 1 al 8), CA1 (organización C, Administradora del hogar 1) y DA 1 (organización D, Administradora del hogar 1).

Según los hallazgos de la presente investigación, se establece la siguiente discusión sobre la uniformidad de las políticas administrativas y gerenciales de las organizaciones de regiones educativas en Puerto Rico. 
Cuadro 2. Entrevistas a grupos focales y administradores del hogar basados en las subcategorías establecidas

\begin{tabular}{|c|c|c|}
\hline SUBCATEGORÍAS & $\begin{array}{l}\text { PREGUNTAS PARA LOS } \\
\text { GRUPOS FOCALES } \\
\end{array}$ & $\begin{array}{c}\text { PREGUNTAS PARA LOS } \\
\text { ADMINISTRADORES DEL HOGAR }\end{array}$ \\
\hline $\begin{array}{l}\text { Información } \\
\text { sociodemográfica }\end{array}$ & $\begin{array}{l}\text { ¿Cómo ustedes definen el } \\
\text { perfil de las familias que } \\
\text { educan en el hogar? }\end{array}$ & $\begin{array}{l}\text { ¿Cuánto tiempo lleva practicando la } \\
\text { educación en el hogar? } \\
\text { ¿Cuántos miembros componen su } \\
\text { familia? } \\
\text { ¿A qué organización en el hogar } \\
\text { pertenece? } \\
\text { ¿Cuántos hijos han educado o educan en } \\
\text { el hogar? } \\
\text { ¿Cuál es la preparación académica de la } \\
\text { madre, padre o encargado? } \\
\text { ¿Cuál es la religión que practican en el } \\
\text { hogar? } \\
\text { ¿Quién o quienes trabajan en el hogar? }\end{array}$ \\
\hline $\begin{array}{l}\text { Reglamentos de las } \\
\text { organizaciones }\end{array}$ & $\begin{array}{l}¿ C \text { ¿Cuáles son las políticas y } \\
\text { reglamentos por los cuales } \\
\text { se rige su organización? }\end{array}$ & $\begin{array}{l}\text { ¿Usted considera que el DEPR cuenta con } \\
\text { una estructura organizativa para los } \\
\text { educados en el hogar? } \\
¿ \text { ¿Conoce usted las políticas y reglamentos } \\
\text { de la educación en el hogar en Puerto } \\
\text { Rico de la organización a la cual } \\
\text { pertenecen? } \\
\text { ¿Puede mencionar algunas de esas } \\
\text { políticas y reglamentos? }\end{array}$ \\
\hline $\begin{array}{c}\text { Métodos de } \\
\text { educación en el } \\
\text { hogar }\end{array}$ & $\begin{array}{l}\text { ¿Cuál es la metodología que } \\
\text { más utilizan los padres que } \\
\text { pertenecen a esta } \\
\text { organización? }\end{array}$ & $\begin{array}{l}\text { ¿Qué tipo de actividades } \\
\text { extracurriculares lleva a cabo con su } \\
\text { hijo para complementar la fase } \\
\text { educativa? } \\
\text { ¿Qué procedimientos usted lleva a cabo } \\
\text { para evidenciar que su hijo cumple con } \\
\text { los requisitos académicos? }\end{array}$ \\
\hline
\end{tabular}

Fuente: Elaboración propia.

\subsection{El perfil de las familias que educan en el hogar}

De acuerdo con la información que brindaron los directivos y administradores del hogar de las cuatro organizaciones el perfil de las familias que educan en el hogar es variado. En las familias entrevistadas se encontraron desde familias pequeñas hasta familias numerosas. Hay familias donde solamente el padre trabaja y la madre se dedica al hogar, o familias en las que ambos trabajan. Las familias profesan diferentes religiones y el perfil socioeconómico y nivel académico son variados. Cada familia es un centro educativo diferente, con sus particularidades, pero centrado en atender las necesidades académicas y personales de su(s) hijo(s).

De acuerdo con los resultados de las entrevistas colectivas (grupos focales) e individuales, las familias que educan en el hogar promueven en sus hijos valores cristianos y muestran compromiso con la educación de sus hijos. 
Cuadro 3. Resumen comparativo de las organizaciones de educación en el hogar en Puerto Rico

\begin{tabular}{|c|c|c|c|c|c|}
\hline & COMPOSICIÓN FAMILIAR & $\begin{array}{c}\text { PERFIL TUTORES (PADRES, MADRES, } \\
\text { ENCARGADOS) }\end{array}$ & $\begin{array}{c}\text { ATENCIÓN A LAS } \\
\text { NECESIDADES } \\
\end{array}$ & $\begin{array}{l}\text { MeTODOLOGÍAS DE } \\
\text { ENSEÑANZA }\end{array}$ & $\begin{array}{l}\text { GRUPOS DE } \\
\text { APOYO } \\
\end{array}$ \\
\hline A & $\begin{array}{l}\text { Variada, compuesta desde tres } \\
\text { hasta ocho miembros }\end{array}$ & $\begin{array}{l}\text { El perfil es variado, preparación académica } \\
\text { desde grado asociado hasta maestría, en la } \\
\text { mayoría de las familias el padre trabaja }\end{array}$ & $\begin{array}{l}\text { Educación } \\
\text { individualizada } \\
\text { basada en las } \\
\text { necesidades del niño }\end{array}$ & $\begin{array}{l}\text { Montessori } \\
\text { Ecléctico } \\
\text { Moore }\end{array}$ & $\begin{array}{l}\text { Se reúnen } \\
\text { mensualmente }\end{array}$ \\
\hline B & Dos personas (niña y abuela) & $\begin{array}{l}\text { Bachillerato, Lleva } 5 \text { años educando en el } \\
\text { hogar }\end{array}$ & $\begin{array}{l}\text { Enseñanza } \\
\text { individualizada } \\
\text { basada en las } \\
\text { necesidades del niño }\end{array}$ & Ecléctica & $\begin{array}{l}\text { Se reúnen } \\
\text { mensualmente }\end{array}$ \\
\hline $\mathbf{C}$ & Dos personas (niño y madre) & $\begin{array}{l}\text { Bachillerato, Madre no trabaja, Un año } \\
\text { educando en el hogar }\end{array}$ & $\begin{array}{l}\text { Destrezas y } \\
\text { reforzarla hasta que } \\
\text { el estudiante la } \\
\text { domine }\end{array}$ & $\begin{array}{l}\text { Montessori } \\
\text { Ecléctica }\end{array}$ & $\begin{array}{l}\text { Se reúnen } \\
\text { mensualmente }\end{array}$ \\
\hline \multirow[t]{2}{*}{$\mathbf{D}$} & $\begin{array}{l}\text { Compuesta desde } 2 \text { hasta } 5 \\
\text { miembros }\end{array}$ & $\begin{array}{l}\text { Variado, preparación académica desde grado } \\
\text { asociado hasta doctorado, llevan educando en } \\
\text { el hogar desde una semana hasta trece años }\end{array}$ & $\begin{array}{l}\text { Educación } \\
\text { individualizada }\end{array}$ & $\begin{array}{l}\text { Ecléctica } \\
\text { Montessori }\end{array}$ & $\begin{array}{l}\text { Se reúnen } \\
\text { mensualmente }\end{array}$ \\
\hline & MATERIAL EDUCATIVO & ACTIVIDADES EXTRACURRICULARES & TRABAJOS & EVALUACIÓN DE TAREAS & HORARIO \\
\hline A & $\begin{array}{l}\text { Libros, Mapas curriculares, } \\
\text { Manuales, Charlas y talleres } \\
\text { educativos, Pruebas } \\
\text { estandarizadas, Manipulativos }\end{array}$ & $\begin{array}{l}\text { Charlas, Feria Científica, Día de logros, Talent } \\
\text { Show, Excursiones, Grupo de Apoyo, } \\
\text { Talleres, Parques, Museos, Deportes }\end{array}$ & Portafolio & $\begin{array}{l}\text { Exámenes escritos y orales } \\
\text { Pruebas Estand. Learn Aid y } \\
\text { Piense I, Assessment }\end{array}$ & Variado flexible \\
\hline B & $\begin{array}{l}\text { Libros, Internet, Unidades de } \\
\text { estudio, Talleres, Mapas } \\
\text { curriculares }\end{array}$ & $\begin{array}{l}\text { Excursiones, Día de Logros, Día de Juegos, } \\
\text { Experimentos ambientales }\end{array}$ & Portafolio & $\begin{array}{l}\text { Exámenes, Dictados, } \\
\text { Experimentos, Monografías, } \\
\text { Ensayos }\end{array}$ & 5 horas diarias \\
\hline $\mathbf{C}$ & $\begin{array}{l}\text { Libros, Mapas curriculares del } \\
\text { DE, Internet, Manuales }\end{array}$ & $\begin{array}{l}\text { Grupos de apoyo, Museo, Parques, Lugares } \\
\text { históricos }\end{array}$ & Portafolio & Exámenes, Assessment & Variado flexible \\
\hline D & $\begin{array}{l}\text { Libros, Manuales, Talleres, Mapas } \\
\text { curriculares del DE }\end{array}$ & $\begin{array}{l}\text { Grupos de apoyo, Excursiones, Actividades } \\
\text { extracurriculares, Obras de teatro, Deportes }\end{array}$ & Portafolio & $\begin{array}{l}\text { Exámenes, Pruebas estand., } \\
\text { Assessment }\end{array}$ & Variado flexible \\
\hline
\end{tabular}


Cuadro 4. Resumen comparativo de los reglamentos de las organizaciones de educación en el hogar

\begin{tabular}{|c|c|c|c|c|}
\hline ARTíCULOS & ORGANIZACIÓN A & ORGANIZACIÓN B & ORGANIZACIÓN C & ORGANIZACIÓN D \\
\hline I & $\begin{array}{l}\text { Establece el propósito de la } \\
\text { Organización }\end{array}$ & Establece el propósito de la Organización & Nombre de la Organización & $\begin{array}{l}\text { Membresía y } \\
\text { responsabilidad de } \\
\text { los participantes }\end{array}$ \\
\hline II & $\begin{array}{l}\text { Membresía (elegibilidad y } \\
\text { membresía) }\end{array}$ & Membresía (elegibilidad y renovación) & Propósito, visión y misión & Beneficios \\
\hline III & $\begin{array}{l}\text { Junta de Directores } \\
\text { (composición, requisitos, } \\
\text { nominación, término del } \\
\text { cargo, deberes y poderes) }\end{array}$ & $\begin{array}{l}\text { Junta de Directores (composición, requisitos, } \\
\text { nominación, elección, término del cargo, } \\
\text { deberes y poderes, vacantes, renuncias, } \\
\text { destitución y transferencia de records) }\end{array}$ & $\begin{array}{l}\text { Definiciones (posición, decreto de fe y } \\
\text { estándares de conducta) }\end{array}$ & Conducta \\
\hline IV & $\begin{array}{l}\text { Grupos de apoyo (afiliados, } \\
\text { líderes de la organización y } \\
\text { vocal) }\end{array}$ & $\begin{array}{l}\text { Reunión general, anual, quorum y resolución } \\
\text { de asuntos }\end{array}$ & $\begin{array}{l}\text { Membresía (vigencia, comportamiento, } \\
\text { participación y coordinador de } \\
\text { actividades) }\end{array}$ & Actividades \\
\hline $\mathrm{V}$ & Comités (Comisión Especial) & Comités & Membresía & Enmiendas \\
\hline VI & $\begin{array}{l}\text { Asamblea anual y Resolución } \\
\text { de asuntos }\end{array}$ & $\begin{array}{l}\text { Año fiscal, contrato, préstamos, depósitos y } \\
\text { deudas }\end{array}$ & $\begin{array}{l}\text { Asambleas (extraordinarias, quorum, } \\
\text { reuniones ordinarias y procedimientos } \\
\text { para votar) }\end{array}$ & $\begin{array}{l}\text { Aceptación de normas } \\
\text { de procedimientos }\end{array}$ \\
\hline VII & $\begin{array}{l}\text { Año fiscal, contratos, } \\
\text { préstamos, depósitos y deudas }\end{array}$ & Libros, expedientes e inspecciones & $\begin{array}{l}\text { Junta Permanente (cualificación, } \\
\text { selección, poderes, vacantes y reuniones) }\end{array}$ & \\
\hline VIII & $\begin{array}{l}\text { Libros, expedientes e } \\
\text { inspecciones }\end{array}$ & Disolución & $\begin{array}{l}\text { Junta Directiva (cualificación. Términos, } \\
\text { destitución, vacante, poderes, reuniones, } \\
\text { informes, transparencia en los } \\
\text { procedimientos) }\end{array}$ & \\
\hline IX & Enmiendas & Enmiendas & $\begin{array}{l}\text { Organización y tareas de la Junta } \\
\text { Directiva }\end{array}$ & \\
\hline $\mathrm{X}$ & & & Recaudación de fondos & \\
\hline $\mathrm{XI}$ & & & Disposiciones misceláneas & \\
\hline
\end{tabular}

Fuente: Elaboración propia. 
Aunque tienen modalidades diferentes de educar a sus hijos, consideran las necesidades académicas particulares de cada niño. Los resultados de la presente investigación apoyan, además, los resultados encontrados por Urpí (2010). Este señaló en su investigación que la educación en el hogar en Estados Unidos, desde sus inicios, está asociada a grupos sociales opuestos: hippies, ecologistas, conservadores y acomodados. Según este autor, cada metodología utilizada para la educación en el hogar es variable y se debe al número de hijos educados en el hogar y a la ocupación e intereses de los padres. Además, Ray (2016) en su investigación señaló que la educación en el hogar en los países de Canadá, Australia, Francia, Hungría, Japón, Kenia, Rusia, Tailandia y Reino Unido, entre otros países, posee una amplia variedad demográfica, tal como: ateos, cristianos, mormones, conservadores, liberales, familias con bajos y altos ingresos, negros, hispanos y padres con doctorados o sin preparación académica. En adición, encontró que el perfil sociodemográfico de las familias que educan en el hogar es variado y que depende de diversas razones por las cuales las familias determinan cual es la mejor educación para sus hijos.

\subsection{Políticas administrativas y gerenciales de las organizaciones de educación en el hogar}

De acuerdo con los resultados de la presente investigación, un grupo focal y una presidenta respondieron que conocen las políticas y normativas por las cuales se rige la organización a la que pertenecen e hicieron mención de las mismas. Cabe resaltar que la mayoría de las educadoras del hogar se abstuvieron de mencionar las políticas normativas de la organización a la cual pertenecen. Otro aspecto que mencionaron las administradoras del hogar participantes de la presente investigación fue que las organizaciones de educación en el hogar en Puerto Rico son sin fines de lucro y tienen reglamentos internos. También indicaron que las organizaciones no necesitan de políticas fiscalizadoras y que su objetivo es garantizarle el tiempo de estudio de sus hijos, ofrecer ayuda individualizada y fomentar el proceso de socialización mediante actividades extracurriculares.

Rodríguez (2013) afirmó que la educación en el hogar en Puerto Rico no necesita regulaciones ni notificaciones del Departamento de educación de Puerto Rico. Cada hogar es una entidad no gubernamental y el éxito de la educación en el hogar depende de la postura que asuman los padres como educadores. Este planteamiento apoya la presente investigación ya que encontró que la educación en el hogar en Puerto Rico no es regulada ya que se fundamenta en el Artículo II Sección 5 de la Carta de Derechos de la Constitución de Puerto Rico (1952). Otro aspecto que Rodríguez (2013) apoya es que cada hogar es una entidad no gubernamental y el éxito de la educación en el hogar depende de la seriedad y postura que los padres asuman en su rol como educadores. También argumenta que la educación en el hogar es una entidad donde los estudiantes socializan mejor y que es ahí donde se enseñan los valores que ese individuo necesita para relacionarse con otras personas.

\subsection{Comparación de las políticas administrativas y gerenciales de las organizaciones de educación en el hogar}

$\mathrm{Al}$ analizar los reglamentos y políticas normativas de las organizaciones que participaron de la investigación, la investigadora encontró que son similares (organización A, B y C). En cada artículo se establecen los parámetros por los que se rige su organización. La Junta Directiva es fundamental en las tres organizaciones, es la que 


\section{G. L. García Bonilla}

diariamente lucha por los derechos de las familias que educan en el hogar. Cada organización establece la cantidad de miembros de la Junta Directiva y establecen sus funciones. Tienen establecidas las fechas para el pago de las cuotas y el calendario para las actividades magnas, como lo son: el Día de Logros, Talent Show, Ferias Científicas, entre otros.

Otro aspecto similar es que establecen el propósito como institución educativa, las familias tienen la libertad para seleccionar la organización de educación en el hogar en la que encuentren mayor afinidad con sus creencias y valores. Anualmente, realizan asambleas para determinar aspectos importantes de las organizaciones en temas relacionados a la educación en el hogar. Aunque no está escrito en todos los reglamentos, cada organización tiene su grupo de apoyo por área y se reúnen mensualmente. La investigadora fue partícipe de esas reuniones en todas las organizaciones. Estas organizaciones no se oponen a la regulación, sino a la fiscalización por parte del gobierno de Puerto Rico.

Los hallazgos de la presente investigación coinciden con el proyecto de Ley 2754 de Rivera (2006). La exrepresentante, en su proyecto, le daba la oportunidad al padre, tutor o encargado para que formara parte de las diferentes organizaciones y grupos de apoyo que existían para que se le facilitara el proceso de educar en el hogar. Sin embargo, hay otros aspectos que no guardan relación entre los reglamentos de las organizaciones. Por ejemplo, en las organizaciones A, B y C incluyen las Enmiendas al reglamento y el Comité Especial para tratar los asuntos legales. Solamente la organización B trabaja la disolución de la organización y los procesos a seguir si llegase a suceder. La organización C es la única que establece el nombre de la organización, define los términos relacionados a la educación en el hogar y cómo se lleva a cabo la recaudación de fondos dentro de la organización. Es importante señalar que la organización C no defiende a las familias que deciden educar en el hogar en un pleito legal ni certifica que el niño recibe este tipo de educación. Por último, solo la organización D establece los beneficios que reciben las familias al ser parte de la misma y las actividades que llevan a cabo para desarrollar en todos sus miembros el proceso de socialización.

Los hallazgos de la presente investigación no concuerdan con los encontrados por Stewart y Neeley (2005) debido a que en Puerto Rico no existen regulaciones de parte del estado para regular la educación en el hogar. Estos encontraron que no había consistencia en las regulaciones de los estados con relación a las reglamentaciones de la educación en el hogar. Sin embargo, en Puerto Rico falta información estadística sobre la cantidad de estudiantes que son educados en el hogar. Cada familia que educa en el hogar se afilia a una organización que seleccionan a base de sus principios religiosos, éticos y educativos, entre otros. Actualmente no existe regulación en torno a educar en el hogar. Sin embargo, cada organización posee un reglamento interno.

\subsection{Metodologías de enseñanza}

Cada hogar es un escenario educativo diferente basado en las necesidades particulares de cada niño. Las familias utilizan una diversidad de metodologías de enseñanza al impartir las clases diariamente. La diversidad de tareas y actividades complementarias son evidenciadas en portafolios para demostrar la ejecución del estudiante. Otra manera de demostrar el aprendizaje adquirido por los estudiantes son las pruebas estandarizadas y el College board. Las actividades extracurriculares (deportes, ferias científicas, excursiones, estudio de campo, día de logros, entre otros) contribuyen a que la educación 
en el hogar se fundamente en un currículo flexible (exámenes, uso de assessment e integración de la tecnología), innovador, individualizado y pertinente a la vida diaria, donde cada niño desarrolle al máximo sus habilidades y talentos. Cada grupo de apoyo establece las actividades que llevará a cabo para que las familias participen, además fortalecen el proceso de socialización entre los niños. La diferencia en metodología y la flexibilidad de horario y currículo es lo que hace que la educación en el hogar en Puerto Rico sea una modalidad educativa innovadora y aceptada por muchas familias.

Los hallazgos de la presente investigación coinciden con los planteamientos de Urpí (2010). En la presente investigación se encontró que cada familia utiliza una variedad de metodologías y técnicas de enseñanza para impartir las clases. Urpí (2010) encontró que no todas las familias que educan en el hogar concuerdan con la misma metodología. Las familias utilizan diferentes estilos, unos más dirigidos que otros. Además, las familias seleccionan los currículos, ya sea el currículo del estado, crear su propio currículo o utilizar libros de texto.

\section{Conclusiones}

Luego de analizar los resultados presentados y teniendo como base la pregunta de investigación, se puede concluir que no existe uniformidad en las políticas administrativas y gerenciales en las organizaciones de educación en el hogar en Puerto Rico. La educación en el hogar está liderada por las féminas, madres de familia con diferente preparación académica y nivel socioeconómico. En adición, los Directivos de las organizaciones conocen y practican los reglamentos y regulaciones por los cuales se rige su organización, sin embargo, muchas administradoras del hogar se abstuvieron a contestar la pregunta. También se puede concluir que el utilizar diferentes metodologías de enseñanza y atender las necesidades particulares de cada niño hace de la educación una flexible y atractiva.

La presente investigación se centró en tres regiones educativas, por lo tanto, los datos obtenidos no se pueden generalizar. Por tal motivo, una de las recomendaciones es replicar esta investigación en las demás regiones educativas en Puerto Rico para realizar una comparación con los datos obtenidos. En el futuro se puede añadir como variable la percepción de los niños educados en el hogar. Otro estudio factible es comparar si hay un efecto entre la preparación académica de los padres y las prácticas de educación en el hogar. Es sumamente importante que se estudie y se establezcan los datos estadísticos sobre la cantidad de los estudiantes educados en el hogar que ingresan a la Universidad y que áreas académicas deciden estudiar. La investigación tuvo como limitación que solamente participaron dos Directivos de las cuatro organizaciones en el hogar bajo estudio. El temor de los padres a ser fiscalizados por el gobierno por educar en el hogar no permitió respuestas amplias y con detalles.

\section{Aportación de la investigación}

Los resultados de esta investigación permitieron desarrollar un modelo de un reglamento para uniformar los procesos gerenciales de las organizaciones que agrupan a las familias que educan en el hogar en Puerto Rico.

El modelo de la figura 2 representa un esquema sugerido para el Manual de procedimientos uniformes para las organizaciones de regiones educativas en Puerto Rico. Con el mismo se pretende dar uniformidad a los procesos gerenciales de cada 
organización. Luego de estudiar y analizar los reglamentos de las organizaciones bajo estudio, la investigadora determinó diecisiete elementos importantes que deben estar presentes en un reglamento.

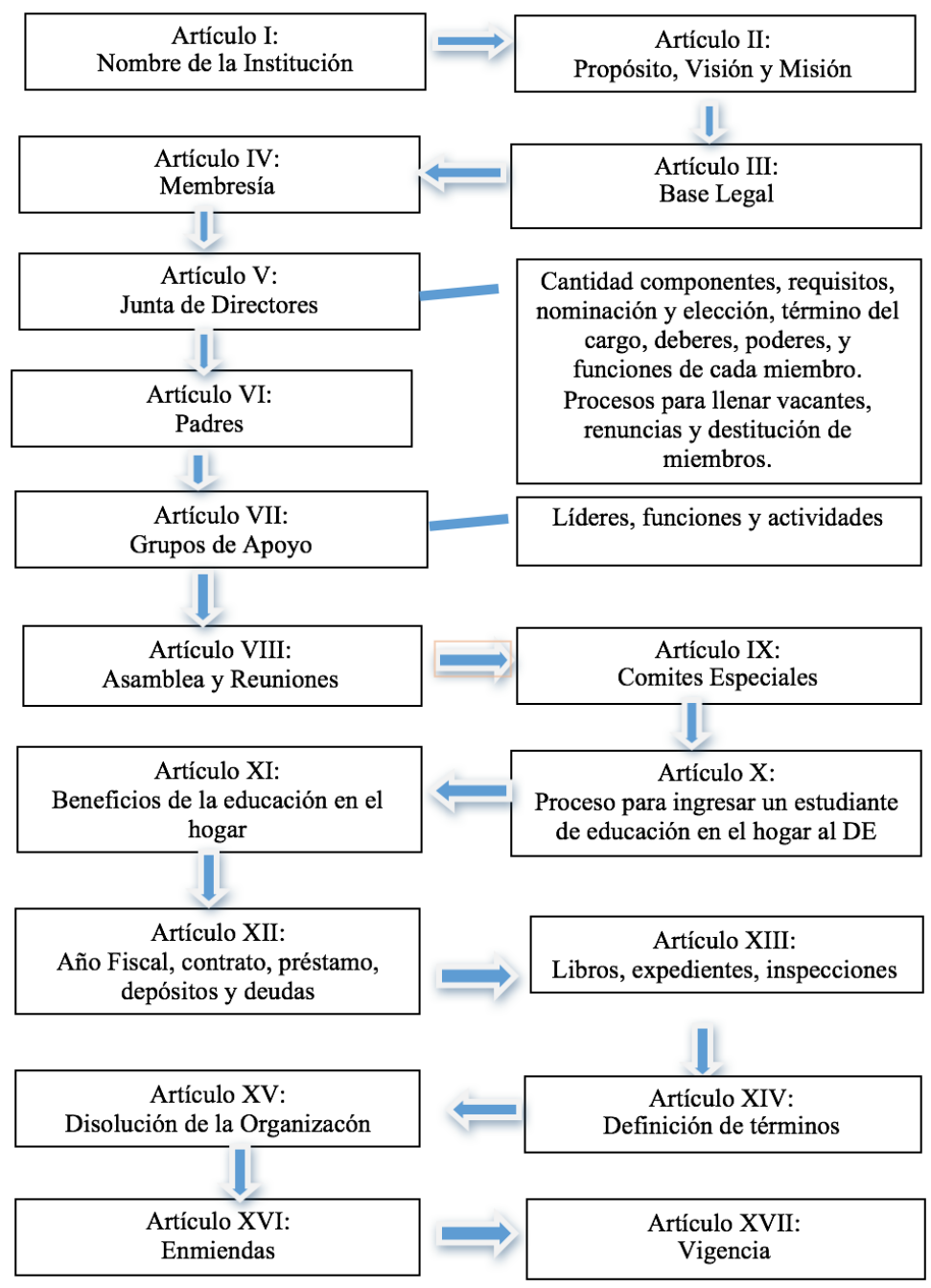

Figura 2. Modelo sugerido: Manual para uniformar los procesos gerenciales de las organizaciones que agrupan a las familias que educan en el hogar

Fuente: Elaboración propia.

En el Artículo I es sumamente importante indicar el nombre de la organización y cuándo fue inscrita en el Departamento de Estado de Puerto Rico, lo cual da mayor seguridad y confiabilidad a las familias al momento de buscar información para afiliarse en una organización.

El Artículo II trata el propósito, visión y misión. Es uno de los artículos de mayor peso porque determina lo que es la organización, qué aspira ser, hacia dónde se dirige y qué desea lograr. Los padres deben conocer su propósito para ser objetivos y poder seleccionar la mejor organización.

En el Artículo III se sugiere que se establezca la base legal. Son los principios legales por los cuales se fundamenta la organización y las leyes que los protegen. De esta manera las 
familias se sentirán más seguras al momento de seleccionar la organización a la que desean afiliarse.

En el Artículo IV se establece la membresía. Es importante establecer la cuota de afiliación, el año fiscal, cuándo se debe renovar y los requisitos mínimos para pertenecer a la organización. Se recomienda que el año fiscal sea igual al año escolar académico en curso, según lo establece el Departamento de Educación de Puerto Rico, para evitar confusiones con relación a cuándo se debe renovar la membresía. Otra recomendación es establecer las cuotas de las actividades extracurriculares, como lo son el Talent Show, el Día de Logros y las Graduaciones, entre otras.

El Artículo v presenta la Junta de Directivos. Es importante determinar que la cantidad de los componentes sea la misma para todas las organizaciones, al igual que los cargos y las funciones. Se recomiendan los siguientes puestos: Presidente, Vicepresidente, Secretaria, Tesorero, Webmaster y un vocal por cada grupo de apoyo formado. La Junta Directiva debe tener como mínimo seis miembros y hasta un máximo de diez. Cada organización debe ser clara cuando establece los requisitos, el término para ser elegido a algún puesto en la Junta de Directores y cómo se realiza el proceso de elección de los miembros, ya que son la base de la organización. Se recomienda que las nominaciones para la Junta de Directores sean por un término de dos años y se haga una nominación especial de surgir alguna vacante.

El Artículo VI establece las funciones y responsabilidades de los padres dentro de las organizaciones y en el hogar al momento de educar a sus hijos. Es importante que los padres comprendan que su rol es vital dentro de lo que es educar en casa. Se les debe ofrecer talleres de como es el proceso de educar en el hogar y de estrategias al momento de seleccionar el contenido curricular para sus hijos. Además, deben velar por la conducta de sus hijos en cada actividad que se realice y participar de las mismas.

Los grupos de apoyo se consideran en el Artículo VII. Se debe tener claro cómo van a ser elegidos los miembros y el líder de cada grupo de apoyo y cuáles son las funciones y las actividades que van a llevar a cabo los mismos. Es importante que anualmente cada grupo de apoyo prepare un calendario de actividades para evitar la improvisación. Ese plan se debe evaluar al finalizar el año académico.

La realización de las Asambleas, Artículo VIII, es un punto de suma importancia para las organizaciones porque es el momento donde se toman decisiones importantes para las mismas, en consenso con los miembros. Se recomienda que se establezca una cantidad mínima de personas para establecer el quorum, y lo que dependerá de la cantidad de miembros activos de la organización.

El Artículo IX incluye el Comités Especiales que pueden estar compuestos por personal interno y externo a la organización, que aportan significativamente a la misma con sus conocimientos y experiencia en el ámbito educativo puertorriqueño.

El Artículo X establece el proceso para el ingreso de los educados en el hogar al sistema de educación público de Puerto Rico. Este es un aspecto importante que se debe considerar en los reglamentos de las organizaciones, de la misma manera que el Departamento de Educación lo ha establecido para tener uniformidad en los procesos. 


\section{G. L. García Bonilla}

El Artículo XI trabaja los beneficios y ventajas de la educación en el hogar. En este inciso se trabaja con la seguridad de los padres al momento de tomar la decisión de educar en casa, además tienen una visión real de todo lo que pueden lograr con sus hijos.

El Artículo XII establece el año fiscal y todo lo relacionado a contratos, deudas, depósitos y préstamos de la organización y cómo estos van a ser utilizados. Establecer este proceso le brinda al padre confianza y confiabilidad a la organización y a su vez les garantiza la transparencia de los procesos ante las familias.

Los libros, expedientes e inspecciones se destacan en el Artículo XIII. Este Artículo en la organización, de lo que trabaja diariamente la organización y cómo se llevan a cabo esos procesos. Es imprescindible guardar evidencia de cada grupo de apoyo y de las actividades realizadas como organización, además, de los gastos incurridos en cada actividad como Graduaciones, Talent Show, Feria Científica, Día de Logros, entre otros. Esto permite garantizarles a los padres cómo y en qué fue utilizada la cuota de membresía y otras cuotas que pagan.

El Artículo XIV presenta la definición de términos. Los administradores del hogar deben conocer de la fuente primaria y correcta todo lo referente en cuanto a la educación en el hogar, evitando así la improvisación o falta de conocimiento de los padres.

El Artículo XV establece la disolución de la organización. Es importante que las familias conozcan de antemano qué sucederá con su organización si en algún momento tienen que cerrar operaciones. Las familias deberán saber qué sucederá con ellos y con sus hijos.

El Artículo XVI considera las enmiendas a los procesos de la organización. Se debe establecer cada cuanto tiempo se deben realizar las mismas y quiénes tendrán esa responsabilidad. Se recomiendan que mínimo se evalúen anualmente, considerando los cambios educativos del sistema educativo para la toma de decisiones acertadas.

El Artículo XVII considera la vigencia del reglamento. Se recomienda que el reglamento de cada organización debe ser revisado anualmente y cuantas veces será necesario para el bien de la misma.

\section{Referencias}

Acevedo, E. (2012). Criminalidad y violencia en los planteles abona al "homeschooling". Recuperado de http://www.noticel.com/noticia/

Bisquerra, R. (2002). Métodos de la investigación educativa. Barcelona: CEAC

Bronfenbrenner, U. (1979). The ecology of human development. Boston, MA: Harvard University Press.

Cabrera, R. (2010). Investigación cualitativa: Características, métodos y técnicas. Recuperado de: http://www.psicodinamico.es/

Constitución de Puerto Rico. (1952). Carta de derechos a la instrucción pública. Recuperado de: www.lexjuris.com/lexprcont.htm.

Creswell, J. (2009). Research design: Qualitative, quantitative, and mixed methods approaches. Los Ángeles, CA: Sage.

García Cabán, J. J. (2002). Proyecto de la cámara 3048. Recuperado de http://www.hslda.org/hs/state/pr/ 
Holt, J. y Farenga, P. (2003). Teach your own. Lawrence, NY: Pitman Publishing Company.

Home School Legal Defense Association. (2003). La educación en el hogar en Puerto Rico. Recuperado de www.hslda.org/hs/state/pr

Lucca, N. y Berrios, R. (2003). Investigación cualitativa en educación y ciencias sociales. Hato Rey: Publicaciones Puertorriqueñas

Ray, B. (2016). Research facts on homeschooling. Recuperado de www.nheri.org

Rivera, A. (2006). Cámara de representantes de Puerto Rico (proyecto 2754). Educación en el hogar como institución educativa no tradicional. Recuperado de http://www.camaraderepresentantes.org/

Rivera Schatz, T. (2011). Proyecto 2110. Ley del derecho de la educación en el hogar. Recuperado de http://www.senadopr.us/Proyectos\%20del\%20Senado/ps2 110-11.pdf

Rivero, L. (2008). The homeschooling option. How to decide when it's right for your family. Nueva York, NY: Palgrave Macmillan. https://doi.org/10.1057/9780230610897

Rodríguez, J. (2013). Derrocan tabúes del homeschooling. Recuperado de http://www.periodicolaperla.com/homeschooling

Román, R. (2015). Procedimientos a seguir en los casos de estudiantes provenientes de la alternativa educativa de enseñanza en el hogar. Recuperado de http://intraedu.dde.pr/

Sánchez, J. (2013). Entrevista sobre el homeschooling en Puerto Rico. Recuperado de https://www.homeschoolingcatolico.org/

Stewart, K. y Neeley, R. (2005). The impact of home schooling regulations on educational enrollments in the United States. Education, 126(2), 353-363.

Tirado Rivera, C. (2013). Proyecto del senado 793 para reglamentar el sistema educativo conocido como "homeschooling" o educación en casa en el estado libre asociado de Puerto Rico. Recuperado de http://www.oslpr.org/2013

Urpí, C. (2010). Homeschooling: Aprender en casa. Nuestro Tiempo, 1(2), 24-31.

Von Bertalanffy, L. (1968). Significado del análisis del sistema (general system theory). Nueva York, NY: Brazile.

\section{Breve CV de la autora}

Glenda L. García Bonilla

Doctora en Gerencia Educativa de la Pontificia Universidad Católica de Puerto Rico en Ponce. Posee grado de maestría en Currículo y Enseñanza en Química de la misma institución. Desde el 2008 trabaja para el Departamento de Educación de Puerto Rico, en el cual se ha desempeñado como maestra de Ciencia General de la Escuela Dr. Máximo Donoso Sánchez y como Facilitadora Docente del Programa de Ciencia del Distrito Escolar de Ponce. ORCID ID: 0000-0003-2395-9743. Email: glendagarcia@pucpr.edu 